\title{
COMPLETE SPACE-LIKE HYPERSURFACES WITH CONSTANT MEAN CURVATURE IN A LORENTZ SPACE FORM OF DIMENSION 4
}

\author{
By Reiko Aiyama And Qing-Ming Cheng*
}

\begin{abstract}
On complete space-like hypersurfaces with constant mean curvature in a Lorentz space form of dimension 4 , we study the case that the scalar curvature is constant and that the Ricci curvature is bounded from above.
\end{abstract}

\section{Introduction.}

Let $\boldsymbol{R}_{1}^{n+1}$ be an $(n+1)$-dimensional Minkowski space and $\boldsymbol{S}_{1}^{n+1}(c)\left(\right.$ resp. $\left.\boldsymbol{H}_{1}^{n+1}(c)\right)$ be an $(n+1)$-dimensional de Sitter space (resp. anti-de Sitter space) of constant curvature $c$. Considered collectively, a Lorentz manifold of these kinds is called a Lorentz space form of constant curvature $c$, which is denoted by $M_{1}^{n+1}(c)$.

Since Calabi [4] and S. Y. Cheng and Yau [7] proved the Bernstein type theorem in $\boldsymbol{R}_{1}^{n+1}$, complete space-like hypersurfaces with constant mean curvature in a Lorentz space form $M_{1}^{n+1}(c)$ have been studying by many mathematicians. On the other hand, space-like hypersurfaces with constant mean curvature in spacetimes get interested in relativity theory.

It is well known that totally umbilical hypersurfaces $M^{n}\left(c^{\prime}\right)\left(c^{\prime}<c\right)$ and hypersurfaces in the form of $\boldsymbol{H}^{k}\left(c_{1}\right) \times M^{n-k}\left(c_{2}\right)\left[k=1, \cdots, n-1, c_{1}<0, c\left(c_{1}+c_{2}\right)=c_{1} c_{2}\right]$ are standard models of complete space-like hypersurfaces with constant mean curvature in $M_{1}^{n+1}(c)$. Here $M^{n}(c)$ means an $n$-dimensional space form with constant curvature $c$, that is, a Riemannian sphere $\boldsymbol{S}^{n}(c)$, a hyperbolic space $\boldsymbol{H}^{n}(c)$ or a Euclidean space $\boldsymbol{R}^{n}$.

Let $M$ be a complete space-like hypersurface with constant mean curvature $h / n$ in $M_{1}^{n+1}(c)$. In a de Sitter space $\boldsymbol{S}_{1}^{n+1}(c), M$ is nothing but totally umbilical if $n=2$ and $h^{2} \leqq 4 c$ or if $n>2$ and $h^{2}<4(n-1) c$ (cf. Akutagawa [3], Ramanathan [12] or Cheng [5]).

In the other case, there are many examples in $M_{1}^{n+1}(c)$ which are not standard models (cf. Treibergs [13], Ishihara and Hara [8], Akutagawa [3] and others). But we have known some characterizations of standard models with respect to

* The Research Supported by National Natural Science Foundation of China.

Received November 7, 1991; revised March 23, 1992. 
the squared norm $S$ of the second fundamental form $\alpha$ on $M$. We define numbers $S_{0}, S_{-}$and $S_{+}$by $S_{0}=h^{2} / n$ and $S_{ \pm}=-n c+\left\{n h^{2} \pm(n-2) \sqrt{h^{4}-4(n-1) c h^{2}}\right\} /$ $2(n-1)$, respectively. Then $S_{0} \leqq S_{-} \leqq S_{+}$. In [9], Ki, Kim and Nakagawa proved that

$$
S_{0} \leqq S \leqq S_{+},
$$

where $S \equiv S_{+} \neq S_{0}$ only when $M$ is a hyperbolic cylinder $\boldsymbol{H}^{1}\left(c_{1}\right) \times M^{n-1}\left(c_{2}\right)$. Also we remark that $S \equiv S_{0}$ only when $M$ is a totally umbilical hypersurface $M^{n}\left(c^{\prime}\right)$ in $M_{1}^{n+1}(c)$. Furthermore, in a de Sitter space $S_{1}^{n+1}(c)$, the second author and Nakagawa [6] proved that if $h^{2} \leqq n^{2} c$ and $\sup S<S_{-}$then $M$ is nothing but a totally umbilical hypersurface.

In the case of $n=2$, the hyperbolic cylinder $\boldsymbol{H}^{1}\left(c_{1}\right) \times M^{1}\left(c_{2}\right)$ is the only complete space-like surface in $M_{1}^{3}(c)$ with constant mean curvature $h / 2$ on which $S$ satisfies $\inf S>S_{0}$ (cf. Aiyama [2]).

However, in the case of $n=3$, we have an example on which $S$ is constant and satisfies $S_{0}<S<S_{+}$, that is, $\boldsymbol{H}^{2}\left(c_{1}\right) \times M^{1}\left(c_{2}\right)$ in $M_{1}^{4}(c)(c \geqq 0)$ satisfies $S \equiv S_{-}$. So the first purpose of this paper is to study the 3-dimensional complete spacelike hypersurfaces with constant mean curvature and constant $S$ in Lorentz space forms.

THEOREM 3.1. Let $M$ be a complete space-like hypersurface with non-zero constant mean curvature and constant scalar curvature in $M_{1}^{4}(c)$. If $S>S_{-}$then $M$ is nothing but a hyperbolic cylinder.

In particular, we can completely classify complete space-like hypersurfaces with constant mean curvature and constant scalar curvature in $S_{1}^{4}(c)$ if $h^{2} \leqq 9 c$ (Theorem 3.2).

The paper is organized as follows. In Section 2 we give the basic concepts and prove some local formulae. In Section 3 we study the 3-dimensional complete space-like hypersurfaces with constant mean curvature and constant scalar curvature, and prove Theorem 3.1 and Theorem 3.2. At last, in Section 4 we consider the case that the Ricci curvature is bounded from above by $3\left(c-h^{2} / n^{2}\right)$.

Authors would like to thank Professor Hisao Nakagawa for his advice and encouragement.

\section{Local formulae.}

Throughout this paper, we assume manifolds to be connected and geometric objects to be smooth.

Let $(M, g)$ be a space-like hypersurface in an $(n+1)$-dimensional Lorentz space form $M_{1}^{n+1}(c)$. We choose a local field of orthonormal frames $e_{1}, \cdots, e_{n}$ on $M$ adapted to the Riemannian metric $g$ induced from the indefinite Riemannian metric on the ambient space, and $\omega_{1}, \cdots, \omega_{n}$ denote the dual coframes on $M$. The connection forms $\omega_{i}$, are characterized by the structure equations 


$$
\left\{\begin{array}{l}
d \omega_{i}+\sum \omega_{i \jmath} \wedge \omega_{\jmath}=0, \quad \omega_{i j}+\omega_{j i}=0, \\
d \omega_{i j}+\Sigma \omega_{i k} \wedge \omega_{k \jmath}=\Omega_{\imath \jmath}, \\
\Omega_{\imath \jmath}=-1 / 2 \sum R_{\imath j k l} \omega_{k} \wedge \omega_{l},
\end{array}\right.
$$

where $\Omega_{\imath \jmath}$ (resp. $R_{\imath j k l}$ ) denotes the Riemannian curvature form (resp. the components of the Riemannian curvature tensor) of $M$. The second fundamental form $\alpha$ and the mean curvature $H$ of $M$ are denoted by

$$
\alpha=-\sum h_{\imath j} \omega_{i} \omega_{j} e_{0}, \text { and } n H=\sum h_{i i}=h,
$$

respectively. Since $\alpha$ is symmetric tensor,

$$
h_{\imath \jmath}=h_{j i} .
$$

If we think about hypersurfaces with constant mean curvature $H$, we may assume that $H$ is non-negative.

The Gauss equation, the Codazzi equation and the Ricci formulae for the second fundamental form and its covariant derivatives are given by

$$
\begin{aligned}
& R_{\imath j k l}=c\left(\boldsymbol{\delta}_{i l} \boldsymbol{\delta}_{j k}-\boldsymbol{\delta}_{i k} \boldsymbol{\delta}_{j l}\right)-\left(h_{i l} h_{j k}-h_{i k} h_{j l}\right), \\
& h_{\imath j k}-h_{i k \jmath}=0, \\
& h_{\imath j k l}-h_{\imath j l k}=-\sum h_{m j} R_{m i k l}-\sum h_{\imath m} R_{m j k l}, \\
& h_{i j k l m}-h_{\imath j k m l}=-\sum h_{t j k} R_{t i l m}-\sum h_{i t k} R_{t j l m}-\sum h_{i j t} R_{t k l m},
\end{aligned}
$$

where $h_{\imath j k}, h_{\imath j k l}$ and $h_{\imath j k l m}$ denote the components of the covariant derivatives $\nabla \alpha, \nabla \nabla \alpha$ and $\nabla^{3} \alpha$ of $\alpha$, respectively.

The components of the Ricci curvature Ric and the scalar curvature $r$ are given by

$$
\begin{aligned}
& R_{\imath \jmath}=(n-1) c \delta_{i j}-h h_{\imath j}+\sum_{k} h_{\imath k} h_{k \jmath}, \\
& r=n(n-1) c-h^{2}+\sum_{\imath, \jmath} h_{\imath \jmath}{ }^{2} .
\end{aligned}
$$

Now we compute some local formulae under the assumption that the mean curvature of $M$ is constant. For arbitrary fixed point $p$ in $M$ we choose a local frame field $e_{1}, \cdots, e_{n}$ such that

$$
h_{\imath \jmath}=\lambda_{i} \delta_{i \jmath} .
$$

We define functions $S$ and $f_{k}$ as follows:

$$
S=|\alpha|^{2}=\sum \lambda_{l}^{2}, \quad f_{k}=\sum \lambda_{j}^{k} .
$$

The Laplacians of these functions and $|\nabla \alpha|^{2}$ are calculated by using suitably the equations (2.1)-(2.5). 
Then we have the following equations:

$$
\begin{aligned}
& \frac{1}{2} \Delta S=|\nabla \alpha|^{2}+S(S+n c)-h f_{3}-c h^{2}, \\
& \frac{1}{2} \Delta|\nabla \alpha|^{2}=|\nabla \nabla \alpha|^{2}+\{S+(2 n+3) c\}|\nabla \alpha|^{2}+\frac{3}{2}|\nabla S|^{2} \\
& -3 h \sum \lambda_{i} h_{\imath j k}{ }^{2}+3\left[\sum \lambda_{\imath}{ }^{2} h_{i j k}{ }^{2}-2 \sum \lambda_{i} \lambda_{j} h_{\imath j k}{ }^{2}\right], \\
& \frac{1}{3} \Delta f_{3}=-h f_{4}+(S+n c) f_{3}-c h S+2 \sum \lambda_{i} h_{\imath j k}{ }^{2}, \\
& \frac{1}{4} \Delta f_{4}=-h f_{5}+(S+n c) f_{4}-c h f_{3}+2 \sum \lambda_{\imath}{ }^{2} h_{\imath j k}{ }^{2}+\sum \lambda_{i} \lambda_{j} h_{i j k}{ }^{2} .
\end{aligned}
$$

Next we only consider the case that $n=3$. In this case, the functions $f_{4}$ and $f_{5}$ are described by $f_{3}$ as follows:

$$
\begin{aligned}
& f_{4}=\frac{1}{6} h^{4}+\frac{4}{3} h f_{3}+\frac{1}{2} S^{2}-h^{2} S, \\
& f_{5}=\frac{5}{6}\left(S+h^{2}\right) f_{3}+\frac{1}{6} h^{5}-\frac{5}{6} h^{3} S .
\end{aligned}
$$

Now we define functions $\mu_{\imath}(i=1,2,3)$ as $\mu_{i}=\lambda_{i}-H$. So we have

$$
\begin{aligned}
& \mu_{1}+\mu_{2}+\mu_{3}=0, \\
& \left(\mu_{1}\right)^{2}+\left(\mu_{2}\right)^{2}+\left(\mu_{3}\right)^{2}=S-\frac{h^{2}}{3}, \\
& B_{3} \equiv\left(\mu_{1}\right)^{3}+\left(\mu_{2}\right)^{3}+\left(\mu_{3}\right)^{3}=f_{3}-h S+\frac{2}{9} h^{3} .
\end{aligned}
$$

Next, assuming that $S$ is constant, we get the following useful equations.

Proposition 2.1. Let $M$ be a 3-dimensional space-like hypersurface in a Lorentz space form $M_{1}^{4}(c)$ with constant mean curvature $H=h / 3$. If $S$ is constant, then we have

$$
\begin{aligned}
|\nabla \alpha|^{2}= & h B_{3}-S^{2}+\left(h^{2}-3 c\right) S+c h^{2}-\frac{2}{9} h^{4}, \\
|\nabla \nabla \alpha|^{2}= & -\frac{h}{2} \Delta B_{3}+\frac{5}{3} \sum\left(\mu_{i}+\mu_{j}+\mu_{k}\right)^{2} h_{i j k}{ }^{2} \\
& +11 h \Sigma \mu_{i} h_{\imath j k}{ }^{2}+3\left(S-S_{0}\right)\left(S-S_{-}\right)\left(S-S_{+}\right) .
\end{aligned}
$$

Here,

$$
S_{0}=\frac{h^{2}}{3} \quad \text { and }
$$




$$
S_{ \pm}=-3 c+\frac{3}{4} h^{2} \pm \frac{1}{4} \sqrt{h^{4}-8 c h^{2}}=\frac{h^{2}}{3}+\frac{3}{8}\left(\frac{h}{3} \pm \sqrt{h^{2}-8 c}\right)^{2} .
$$

Proof. It follows from $\Delta S=0$ that the equation (2.8) implies

$$
|\nabla \alpha|^{2}=h f_{3}-S(S+3 c)+c h^{2} .
$$

From this equation (2.19) combined with (2.16), we get the equation (2.17).

Also it follows from $\nabla S=0$ that the equation (2.9) implies

$$
|\nabla \nabla \alpha|^{2}=\frac{1}{2} \Delta|\nabla \alpha|^{2}-(S+9 c)|\nabla \alpha|^{2}+3 h A-3(B-2 C),
$$

where $A=\sum \lambda_{i} h_{\imath j k}{ }^{2}, B=\sum \lambda_{2}{ }^{2} h_{\imath j k}{ }^{2}$ and $C=\sum \lambda_{i} \lambda_{j} h_{\imath j k}{ }^{2}$. implies

First we remark that replacing $\lambda_{2}$ in the functions $A, B$ and $C$ with $\mu_{2}$

$$
\begin{aligned}
& A=\sum \mu_{i} h_{\imath j k}{ }^{2}+\frac{h}{3}|\nabla \alpha|^{2}, \\
& B+2 C=\frac{1}{3} \Sigma\left(\mu_{i}+\mu_{j}+\mu_{k}\right)^{2} h_{\imath j k}{ }^{2}+2 h \sum \mu_{i} h_{\imath j k}{ }^{2}+\frac{h^{2}}{3}|\nabla \alpha|^{2} .
\end{aligned}
$$

On the other hand, from the relations (2.10) and (2.12), we can describe the function $A$ with $f_{3}$ :

$$
6 A=\Delta f_{3}-3\left(S-\frac{4}{3} h^{2}+3 c\right) f_{3}+\frac{3}{2} h S^{2}+3 h\left(c-h^{2}\right) S+\frac{1}{2} h^{5} .
$$

From this equation combined with (2.19), we get

$$
2 h A=\frac{1}{3} \Delta|\nabla \alpha|^{2}-\frac{1}{3}\left(3 S-4 h^{2}+9 c\right)|\nabla \alpha|^{2}-\left(S-S_{0}\right)\left(S-S_{-}\right)\left(S-S_{+}\right) .
$$

Also it follows from the equation (2.11) combined with (2.12), (2.13) and (2.19) that we have

$$
\begin{aligned}
2 B+C= & \frac{1}{4} \Delta f_{4}+h f_{5}-(S+3 c) f_{4}+c h f_{3} \\
= & \frac{1}{3} h \Delta f_{3}-\frac{1}{6}\left(3 S-5 h^{2}+18 c\right) h f_{3} \\
& -\frac{1}{6}\left[3(S+3 c)\left(S^{2}-2 h^{2} S+h^{4} / 3\right)+h^{4}\left(5 S-h^{2}\right)\right] \\
= & \frac{1}{3} \Delta|\nabla \alpha|^{2}-\frac{1}{6}\left(3 S-5 h^{2}+18 c\right)|\nabla \alpha|^{2}-\left(S-S_{0}\right)\left(S-S_{-}\right)\left(S-S_{+}\right) .
\end{aligned}
$$

Then, from the equations (2.22) and (2.24), we get 


$$
\begin{aligned}
3(B-2 C) & =\frac{4}{3} \Delta|\nabla \alpha|^{2}-\left(2 S-\frac{5}{3} h^{2}+12 c\right)|\nabla \alpha|^{2} \\
& -10 h \Sigma \mu_{i} h_{i j k}{ }^{2}-\frac{5}{3} \Sigma\left(\mu_{i}+\mu_{j}+\mu_{k}\right)^{2} h_{\imath j k}{ }^{2}-4\left(S-S_{0}\right)\left(S-S_{-}\right)\left(S-S_{+}\right) .
\end{aligned}
$$

At last, computing $|\nabla \nabla \alpha|^{2}$ from (2.20) combined with (2.21), (2.23) and (2.25), we have proved the equation (2.18).

The following generalized maximum principle due to Omori [11] and Yau [14] will play a major part in this paper.

Theorem 2.1 (cf. Omori [11] and Yau [14]). Let $M$ be an n-dimensional complete Riemannian manifold whose Ricci curvature is bounded from below. Let $F$ be a $C^{2}$-function bounded from below on $M$, then for any $\varepsilon>0$ there exists a point $p$ in $M$ such that

$$
F(p)<\inf F+\varepsilon, \quad|\nabla F|(p)<\varepsilon, \quad \Delta F(p)>-\varepsilon .
$$

\section{Proof of Theorems.}

In this section, we consider that $M$ is a 3-dimensional complete space-like hypersurface with constant mean curvature and constant scalar curvature in a Lorentz space form $M_{1}^{4}(c)$ and we prove the theorems stated in the introduction.

For that purpose, we need the proposition below.

Proposition 3.1. Let $M$ be a complete space-like hypersurface with constant mean curvature $H=h / 3$ and constant scalar curvature. Also we define $S$ and $B_{3}$ as in Section 2. Then $S$ is constant, and the function $B_{3}$ satisfies

$$
\left|B_{3}\right| \leqq \frac{1}{\sqrt{6}}\left(S-\frac{h^{2}}{3}\right)^{3 / 2}
$$

When $M$ is not totally umbilical, $B_{3} \equiv \frac{1}{\sqrt{6}}\left(S-\frac{h^{2}}{3}\right)^{3 / 2}$ if and only if $M$ is congruent to a hyperbolic cylinder $\boldsymbol{H}^{1}\left(c_{1}\right) \times M^{2}\left(c_{2}\right)$, and also, $B_{3} \equiv-\frac{1}{\sqrt{6}}\left(S-\frac{h^{2}}{3}\right)^{3 / 2}$ if and only if $M$ is congruent to $\boldsymbol{H}^{2}\left(c_{1}\right) \times M^{1}\left(c_{2}\right)$.

Here we remark that if $M$ has an umbilical point then $M$ is totally umbilicalin in this case.

Proof. According to (2.7), we know that the scalar curvature $r$ is constant if and only if $S$ is constant.

The inequality (3.1) follows from (2.14) and (2.15) by solving the problem 
for the conditional extremum (cf. Okumura [10]), and the equality holds if and only if $\left(\mu_{1}-\mu_{2}\right)\left(\mu_{1}-\mu_{3}\right)\left(\mu_{2}-\mu_{3}\right)=0$. Then the equality $\left|B_{3}\right|=\frac{1}{\sqrt{6}}\left(S-\frac{h^{2}}{3}\right)^{3 / 2}$ means that

$$
\mu_{1}=\mu_{2}= \pm \frac{1}{\sqrt{6}}\left(S-\frac{h^{2}}{3}\right)^{1 / 2} \text { and } \mu_{3}=\mp \frac{2}{\sqrt{6}}\left(S-\frac{h^{2}}{3}\right)^{1 / 2}
$$

except the order. So $\left|B_{3}\right| \equiv \frac{1}{\sqrt{6}}\left(S-\frac{h^{2}}{3}\right)^{3 / 2} \neq 0$ if and only if $M$ has two distinct constant principal curvatures. Therefore this proposition proved by the use of a theorem due to Abe, Koike and Yamaguchi [1].

Here we describe our main theorem and its proof. This theorem characterizes the hyperbolic cylinder $\boldsymbol{H}^{1}\left(c_{1}\right) \times M^{2}\left(c_{2}\right)$ in $M_{1}^{4}(c)$ when the constant mean curvature $h / 3$ of complete space-like hypersurfaces in $M_{1}^{4}(c)$ satisfies $h^{2} \geqq 8 c$. As explained in the introduction, it is known that complete space-like hypersurfaces with constant mean curvature $h / 3$ in $M_{1}^{4}(c)$ are totally umbilical if $h^{2}<8 c$ (cf. Akutagawa [3]). Throughout this section, we assume that $h^{2} \geqq 8 c$. Then we can define real numbers $S_{-}$and $S_{+}$by

$$
S_{ \pm}=\frac{h^{2}}{3}+\frac{3}{8}\left(\frac{h}{3} \pm \sqrt{h^{2}-8 c}\right)^{2} \text {. }
$$

THEOREM 3.1. The hyperbolic cylinder is the only complete space-like hypersurface with constant mean curvature $h / 3$ and constant scalar curvature in $M_{1}^{4}(c)$, whose squared norm $S$ of the second fundamental form is greater than $S_{-}=\frac{h^{2}}{3}+\frac{3}{h}\left(\frac{h}{3}-\sqrt{h^{2}-8 c}\right)^{2}$.

Proof. Since $M$ is not totally umbilical under the assumption $S>S_{-}\left(\geqq S_{0}\right)$, by virtue of Proposition 3.1, it is sufficient to show that $B_{3} \equiv \frac{1}{\sqrt{ } 6}\left(S-\frac{h^{2}}{3}\right)^{3 / 2}$. So we shall prove some contradictions when we assume that inf $B_{3}<$ $\frac{1}{\sqrt{6}}\left(S-\frac{h^{2}}{3}\right)^{3 / 2}$.

The function $B_{3}$ on $M$ is smooth and bounded. Also the formula (2.6) implies that the Ricci curvature of $M$ is bounded from below by $2 c-h^{2} / 4$. These means that Theorem 2.1 can be applied to the function $B_{3}$. Let $\varepsilon$ be any positive number that is small enough to be less than $\frac{1}{\sqrt{6}}\left(S-\frac{h^{2}}{3}\right)^{3 / 2}-\inf B_{3}(>0)$. There exists a point $p$ in $M$, at which $B_{3}$ satisfies the following:

$$
\begin{aligned}
& B_{3}(p)<\inf B_{3}+\varepsilon<\frac{1}{\sqrt{6}}\left(S-\frac{h^{2}}{3}\right)^{3 / 2}, \\
& \left|\nabla B_{3}\right|(p)<\varepsilon, \quad \Delta B_{3}(p)>-\varepsilon .
\end{aligned}
$$


Our proof is divided into the following two cases:

( I ) The case of that $\inf B_{3}=-\frac{1}{\sqrt{6}}\left(S-\frac{h^{2}}{3}\right)^{3 / 2}$,

(II) The case of that $\inf B_{3}>-\frac{1}{\sqrt{6}}\left(S-\frac{h^{2}}{3}\right)^{3 / 2}$.

In the case of (I), it follows from (2.17) and (3.2) that

where

$$
|\nabla \alpha|^{2}(p)<K+h \varepsilon
$$

$$
\begin{aligned}
K= & -\left(S-\frac{h^{2}}{3}\right)\left[\sqrt{S-\frac{h^{2}}{3}}+\frac{3}{2 \sqrt{6}}\left(\frac{h}{3}-\sqrt{h^{2}-8 c}\right)\right] \\
& \times\left[\sqrt{S-\frac{h^{2}}{3}}+\frac{3}{2 \sqrt{6}}\left(\frac{h}{3}+\sqrt{h^{2}-8 c}\right)\right] .
\end{aligned}
$$

Since $S>S_{-}$, we have $K<0$. Accordingly, for an enough small positive number $\varepsilon$, there exists a point $p$ in $M$ such that $|\nabla \alpha|^{2}(p)<0$. This is a contradiction.

Next, we consider the case of (II). In this case, we make use of the equation (2.18) in Proposition 2.1.

Since $h$ and $S$ are constant, at any point $q$ in $M$, we have

$$
\begin{aligned}
& h_{11 k}+h_{22 k}+h_{33 k}=0, \\
& \mu_{1} h_{11 k}+\mu_{2} h_{22 k}+\mu_{3} h_{33 k}=0,
\end{aligned}
$$

where $k=1,2,3$. Also we define the numbers $\delta_{k}(q)(k=1,2,3)$ by

$$
\left[\left(\mu_{1}\right)^{2} h_{11 k}+\left(\mu_{2}\right)^{2} h_{22 k}+\left(\mu_{3}\right)^{2} h_{33 k}\right](q)=\delta_{k}(q) .
$$

From (3.2) and the assumption of (II), we have $\left|B_{3}\right|(p) \leqq \frac{1}{\sqrt{6}}\left(S-\frac{h^{2}}{3}\right)^{3 / 2}$. Then the proof of Proposition 3.1 asserts that $\mu_{1}(p), \mu_{2}(p)$ and $\mu_{3}(p)$ are distinct number with each other. So, when the equations (3.4), (3.5) and (3.6) at $p$ are considered as a system of equations with 3 unknowns $h_{11 k}(p), h_{22 k}(p)$ and $h_{33 k}(p)$, they can be solved uniquely:

$$
h_{i i k}(p)=a^{2}(p) \boldsymbol{\delta}_{k}(p) \quad(i, k=1,2,3) .
$$

Since (2.15) means that the coefficients of the system of equations are bounded, there is a positive number $a$ such that $\left|a^{2}(p)\right|<a(i=1,2,3)$ for any point $p$ in $M$ satisfying (3.2) and (3.3). Furthermore, $\left|\nabla B_{3}\right|(p)<\varepsilon$ in (3.3) implies that $\left|\delta_{k}(p)\right|<\varepsilon$, and also,

$$
h_{i i k}(p)<a \varepsilon \quad(i, k=1,2,3) .
$$

Accordingly, from this (3.7) and (2.14), we have positive constant numbers $K_{1}$ 
and $K_{2}$ such that

$$
\begin{aligned}
& {\left[\sum \mu_{i} h_{\imath j k}{ }^{2}\right](p)<K_{1} \varepsilon^{2},} \\
& {\left[\Sigma\left(\mu_{i}+\mu_{j}+\mu_{k}\right)^{2} h_{\imath j k}{ }^{2}\right](p)<K_{2} \varepsilon^{2} .}
\end{aligned}
$$

Then it follows from (2.18) combined with (3.3) and (3.8) that

$$
|\nabla \nabla \alpha|^{2}(p)<3\left(S-S_{0}\right)\left(S-S_{-}\right)\left(S-S_{+}\right)+\frac{h}{2} \varepsilon+\left(11 h K_{1}+\frac{5}{3} K_{2}\right) \varepsilon^{2} .
$$

Since it is known that $S_{0} \leqq S \leqq S_{+}$by Ki, Kim and Nakagawa [9] and $S>S_{-}$ from the assumption, $\left(S-S_{0}\right)\left(S-S_{-}\right)\left(S-S_{+}\right)$is a negative constant number. Accordingly, for an enough small positive number $\varepsilon$, there exists a point $p$ in $M$ such that $|\nabla \nabla \alpha|^{2}(p)<0$. This is a contradiction, too.

Hence, we get that $B_{3} \equiv \frac{1}{\sqrt{6}}\left(S-\frac{h^{2}}{3}\right)^{3 / 2}$, and complete the proof of Theorem 3.1 .

In a general case, we do not know whether or not there are examples which the squared norm $S$ of the second fundamental form is in the region $S_{0}<S \leqq S_{\text {. }}$. However, when $c>0$ and $h^{2} \leqq 9 c$, we have a nonexistence theorem due to the second author and Nakagawa [6]: Let $M$ be a complete space-like hypersurface with constant mean curvature $H=h / 3$ in $S_{1}^{1}(c)$. If $h^{2} \leqq 9 c$ and $\sup S<S_{-}$, then $M$ is totally umbilical. Furthermore, when $S$ is constant, we can prove nonexistence in the case of which $S \leqq S_{-}$.

PROPOSITION 3.2. There are no complete space-like hypersurfaces with constant mean curvature $H=h / 3$ and constant scalar curvature in $S_{1}^{4}(c)$, on which the squared norm $S$ of second fundamental form satisfies that

(1) $S_{0}<S \leqq S_{-}$if $8 c<h^{2} \leqq 9 c$,

(2) $S_{0}<S<S_{-}\left(=S_{+}\right)$if $h^{2}=8 c$.

Proof. Let $M$ be a complete space-like hypersurface in $\boldsymbol{S}_{1}^{4}(c)$ satisfying the assumption of Proposition 3.2.

It follows from (2.17) combined with (3.1) that we get

$$
\begin{aligned}
|\nabla \alpha|^{2} \leqq & -S^{2}+\left(h^{2}-3 c\right) S+c h^{2}-\frac{2}{9} h^{4}+\frac{h}{\sqrt{6}}\left(S-\frac{h^{2}}{3}\right)^{3 / 2} \\
= & -\left(S-\frac{h^{2}}{3}\right)\left[\sqrt{S-\frac{h^{2}}{3}}-\frac{3}{2 \sqrt{6}}\left(\frac{h}{3}-\sqrt{h^{2}-8 c}\right)\right] \\
& \times\left[\sqrt{S-\frac{h^{2}}{3}}-\frac{3}{2 \sqrt{6}}\left(\frac{h}{3}+\sqrt{h^{2}-8 c}\right)\right] .
\end{aligned}
$$

Hence, we remark that if $h^{2} \leqq 9 c$ then the condition $S \leqq S_{\text {- implies }}$ 


$$
\sqrt{S-\frac{h^{2}}{3}} \leqq \frac{3}{2 \sqrt{6}}\left(\frac{h}{3}-\sqrt{h^{2}-8 c}\right) .
$$

Then the fact $S_{0} \leqq S \leqq S_{+}$means that the right side of the above inequality (3.9) is non-positive. Accordingly, in the inequality (3.9), the equality has to hold. So we get

$$
B_{3} \equiv \frac{1}{\sqrt{6}}\left(S-\frac{h^{2}}{3}\right)^{3 / 2} .
$$

By virtue of Proposition 3.1, this means that $M$ is either a totally umbilical hypersurface or a hyperbolic cylinder. However, the assumption $S_{0}<S<S_{+}$ implies that $M$ is not these standard models by the theorem due to $\mathrm{Ki}$, Kim and Nakagawa [9].

Combined with Theorem 3.1 and Proposition 3.2, we can completely classify complete space-like hypersurfaces with constant mean curvature $H=h / 3$ and constant scalar curvature in $\boldsymbol{S}_{1}^{4}(c)$ if $h^{2} \leqq 9 c$.

THEOREM 3.2. Let $M$ be a complete space-like hypersurface with constant mean curvature $H=h / 3$ and constant scalar curvature in $\boldsymbol{S}_{1}^{4}(c)$. If $h^{2} \leqq 9 c$, then $M$ is congruent to $\boldsymbol{R}^{3}, \boldsymbol{S}^{3}\left(c_{1}\right)$ or a hyperbolic cylinder $\boldsymbol{H}^{1}\left(c_{1}\right) \times \boldsymbol{S}^{2}\left(c_{2}\right)$.

Proof. It follows from Theorem 3.1 and Proposition 3.2 that $M$ must be a totally umbilical hypersurface or a hyperbolic cylinder. In $\boldsymbol{S}_{1}^{4}(c)$, a complete totally umbilical space-like hypersurface is congruent to $\boldsymbol{H}^{3}\left(c_{2}\right), \boldsymbol{R}^{3}$ or $\boldsymbol{S}^{3}\left(c_{1}\right)$. However we can easily check that the hypersurfaces in the form of $\boldsymbol{H}^{3}\left(c_{2}\right)$ do not satisfy the assumption $h^{2} \leqq 9 c$.

\section{Hypersurfaces with Ricci curvature bounded from above.}

In this section we study that $M$ is a 3-dimensional complete space-like hypersurface in a Lorentz space form $M_{1}^{4}(c)$ with constant mean curvature and with Ricci curvature bounded from above.

THEOREM 4.1. The totally umbilical hypersurface $\boldsymbol{S}^{3}\left(c_{1}\right)$ in a de-Sitter space $\boldsymbol{S}_{1}^{4}\left(c_{2}\right)\left(c_{2}>c_{1}>0\right)$ is the only complete space-like hypersurface in a Lorentz space form $M_{1}^{4}(c)$ with constant mean curvature $H$ whose Ricci curvature is bounded from above by some number $\delta$ less than $3\left(c-H^{2}\right)$.

Proof. Let $M$ be a 3-dimensional complete space-like hypersurface with constant mean curvature $H$ in a Lorentz space form $M_{1}^{4}(c)$. Assume that the Ricci curvature is bounded from above by some number $\delta$ less than $3\left(c-H^{2}\right)$.

From (2.8) we have 


$$
\begin{aligned}
\frac{1}{2} \Delta S & =|\nabla \alpha|^{2}+S(S+3 c)-h f_{3}-c h^{2} \\
& =|\nabla \alpha|^{2}+\frac{1}{2} \Sigma\left(c-\lambda_{i} \lambda_{j}\right)\left(\lambda_{2}-\lambda_{j}\right)^{2} \\
& =|\nabla \alpha|^{2}+\frac{1}{2} \sum R_{\imath j j i}\left(\lambda_{2}-\lambda_{j}\right)^{2} .
\end{aligned}
$$

On the other hand, since $M$ is a 3-dimensional submanifold, its Weyl conformal curvature tensor vanishes, i.e.,

$$
R_{\imath j k l}=R_{i l} \delta_{j k}-R_{i k} \delta_{j l}+R_{j k} \delta_{i l}-R_{j l} \delta_{i k}-\frac{r}{2}\left(\delta_{i l} \delta_{j k}-\delta_{i k} \delta_{j l}\right)
$$

Hence we get

$$
R_{\imath j j i}=R_{i i}+R_{j j}-\frac{r}{2}
$$

for any distinct indices $i$ and $j$. By $R_{11}+R_{22}+R_{33}=r$, we have

$$
R_{\imath j j i}=-R_{k k}+\frac{r}{2}
$$

for any distinct indices. Hence we get

$$
\begin{aligned}
\frac{1}{2} \Delta S & =|\nabla \alpha|^{2}+\frac{1}{2} \Sigma\left(\frac{r}{2}-R_{k k}\right)\left(\lambda_{2}-\lambda_{j}\right)^{2} \\
& \geqq \frac{1}{4}(r-2 \delta) \Sigma\left(\lambda_{i}-\lambda_{j}\right)^{2} \\
& =\frac{1}{2}\left(3 S-h^{2}\right)\left(6 c-h^{2}+S-2 \delta\right) \\
& \geqq \frac{1}{2}\left(3 S-h^{2}\right)\left(6 c-\frac{2}{3} h^{2}-2 \delta\right)
\end{aligned}
$$

Applying Theorem 2.1 to the function $F=-S$, we have

$$
0 \geqq \frac{1}{2}\left(3 \sup S-h^{2}\right)\left(6 c-\frac{2}{3} h^{2}-2 \delta\right) \text {. }
$$

Hence $\sup S \leqq(1 / 3) h^{2}$. Thus $M$ is totally umbilical.

On the other hand, the Ricci curvature tensor of a totally umbilical hypersurface $M^{3}\left(c^{\prime}\right)$ in $M_{1}^{4}(c)$ is given by $R_{\imath 3}=2 c^{\prime} \delta_{i 3}=2\left(c-H^{2}\right) \delta_{i 3}$. In order for the totally umbilical hypersurface to satisfy the assumption, $c^{\prime}=c-H^{2}$ must be positive.

We have completed the proof of Theorem 4.1 . 


\title{
REFERENCES
}

[1] K. Abe, N. Kolke and S. Yamaguchi, Congruence theorems for proper semiRiemannian hypersurfaces in a real space form, Yokohama Math. J. 35 (1987), $123-136$.

[2] R. Aiyama, On complete space-like surfaces with constant mean curvature in a Lorentzian 3-space form, Tsukuba J. Math. 15 (1991), 235-247.

[3] K. Akutagawa, On space-like hypersurfaces with constant mean curvature in the de Sitter space, Math. Z. 196 (1989), 3-19.

[4] E. CALABI, Examples of Bernstein problems for some nonlinear equations, Proc. Pure Appl. Math. 15 (1970), 223-230.

[5] Q.M. Cheng, Complete space-like submanifolds in de Sitter space with parallel mean curvature vector, Math. Z. 206 (1991), 333-339.

[6] Q. M. Cheng and H. Nakagawa, Totally umbilical hypersurfaces, Hiroshima J. Math. 20 (1990), 1-10.

[7] S. Y. Cheng AND S.T. YAU, Maximal space-like hypersurfaces in the LorentzMinkowski spaces, Ann. of Math. 104 (1976), 407-419.

[8] T. Ishinara AND F. HARA, Surfaces of revolution in the Lorentzian 3-space, J. Math. Tokushima Univ. 22 (1988), 1-13.

[ 9 ] U.H. KI, H.J. Kim and H. NAKAgawA, On space-like hypersurfaces with constant mean curvature of a Lorentz space form, Tokyo J. Math. 14 (1991), 205216.

[10] M. OKumura, Hypersurfaces and a pinching problem on the second fundamental tensor, Amer. J. Math. 86 (1969), 367-377.

[11] H. OMORI, Isometric immersions of Riemannian manifolds, J. Math. Soc. Japan 19 (1967), 205-214.

[12] J. Ramanathan, Complete spacelike hypersurfaces of constant mean curvature in a de Sitter space, Indiana Univ. Math. J. 36 (1987), 349-359.

[13] A.E. TREIBERGS, Entire hypersurfaces of constant mean curvature in Minkowski 3-space, Invent. Math. 66 (1982), 39-56.

[14] S.T. YAU, Harmonic functions on complete Riemannian manifolds, Comm. Pure and Appl. Math. 28 (1975), 201-208.

\author{
INSTITUTE OF MATHEMATICS, \\ UNIVERSITY OF TSUKUBA, \\ 305 IBARAKI, JAPAN \\ Department of Mathematics, \\ NoRTHEAST UNIVERSITY OF TECHNOLOGY, \\ SHENYANG LIAONING 110006, \\ CHINA
}

